\title{
Bright light treatment an effective addition to antidepressants for non-seasonal depression
}

Martiny K. Adjunctive bright light in non-seasonal major depression. Acta Psychiatr Scand 2004;1 10:7-28.

\section{Does bright white light treatment improve symptoms of non-seasonal depression?}

\section{METHODS}

$\square$

Design: Randomised controlled trial

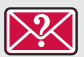

Allocation: Not stated.

Blinding: Single blinded (investigators were blind to group allocation).

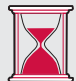

Follow up period: Five weeks.

Setting: General practices in Copenhagen.

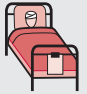

Patients: 107 people with non-seasonal major depressive disorder (DMS-IV); over 18 years old; scoring $>13$ on the Hamilton Depression Scale. Those with seasonal affective disorder, psychotic mental disorder, organic brain disorder, mental retardation, and pregnancy were excluded.

$\mathbf{R}_{\mathbf{X}}$ Intervention: The intervention consisted of five weeks of daily sessions of light treatment in the participant's home. Participants were advised to complete their session before 10:00 each morning, sitting at a distance of $40 \mathrm{~cm}$ with the head directed towards the light. The treatment sessions lasted for one hour a day (intensity: 10000 lux at $40 \mathrm{~cm}$ ) while the placebo involved dim red light for 30 minutes a day (intensity: 50 lux at $40 \mathrm{~cm}$ ). Participants in both groups were also given $50 \mathrm{mg} /$ day of sertraline.

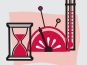

Outcomes: Symptoms of depression, measured on observer rated scales (including the Hamilton Depression Rating Scale $\left(H A M-D_{17}\right)$ and the Hamilton 6-item subscale $\left(H A M-D_{6}\right)$ ), and self-assessment scales (the Major Depression Inventory (MDI) and the Symptom Check List (SCL-90R)). Quality of life measured with the Psychological General Well-Being Scale (PGWB), and the WHO-Five (a subscale of PGWB).

$\square \square$

\section{MAIN RESULTS}

The study found that bright light significantly reduced symptoms of depression on all observer rated scales (HAM-D $D_{17}$ score at 5 weeks: 9.0 with bright light $v 11.6$ with dim light; $\mathrm{p}<0.01, \mathrm{HAM}-\mathrm{D}_{6}$ score at 5 weeks: 5.5 with bright light $v 7.0$ with dim light; $p<0.01)$. The study also found significant reductions at five weeks when depression was self-assessed (change in total score on SCL-90R from baseline: -0.71 with bright light $v-0.49$ with dim light; $\mathrm{p}<0.05$ ). There were significant differences in favour of bright light treatment in the obsessive-compulsive, interpersonal sensitivity, paranoid, and psychoticism dimensions of the scale, while somatisation, depression, anxiety, hostility, and phobic anxiety dimensions were not significantly different between treatments. Quality of life after five weeks

For correspondence: Klaus Martiny, Psychiatric Research Unit, Frederiksborg General Hospital, Dyrehavevej 48, DK-3400 Hilleroed, Denmark; kmar@fa.dk

Sources of funding: not reported.

was not significantly improved in the bright light group (change in PGWB score from baseline: +29.3 with bright light $v+23.3$ with dim light; $\mathrm{p}>0.05$ ). The study also concluded that response to treatment was quicker in the bright light group and that remission was greater. These outcomes were measured on the HAM- $\mathrm{D}_{17}$ scale.

\section{CONCLUSIONS}

For people with non-seasonal depression, bright light is an effective adjunct to antidepressants.

\section{NOTES}

Results that are based on measures using HAM- $\mathrm{D}_{17}$ (response, remission, some observer rated depression results) are questionable as the study was underpowered for the use of this tool.

\section{Commentary}

- vidence supporting bright light's efficacy to treat seasonal depression seems incontrovertible, but data on its efficacy for non-seasonal depression are inconsistent. According to a Cochrane review of randomised controlled trials on the efficacy of bright light, the treatment is generally more effective than placebo when used as an adjunct to pharmacotherapy for non-seasonal depression. However, many of the studies reviewed did not yield significant results. ${ }^{1}$ This observation is echoed in a meta-analysis, finding bright light significantly reduced the severity of depression among patients with seasonal depression $(d=0.73)$ and among those with non-seasonal depression $(d=0.53){ }^{2}$ However, bright light administered concomitantly with antidepressants to treat non-seasonal depression was not as effective $(d=-0.01)$.

Inconsistent results observed from adjunctive bright light studies may be explained by insufficient data, inadequate study designs, and heterogeneity in methodologies. Martiny's study addressed many of the concerns raised in these reviews. Indeed, it is one of a handful of studies showing significant effects of bright light as an adjunctive therapy for non-seasonal depression. The fact that a greater number of people were enrolled and studied over a longer period of time than in previous trials is a particular strength of the current study.

However, the people in the control group received dim light for only 30 minutes, compared with the experimental group who received bright light for one hour-a confounder which the author did not address. Standardisation of all procedures would have contributed to the scientific rigor of the study. Monitoring changes in endogenous melatonin, which were probably commensurate with treatment efficacy, would have been informative. In light of recent data, it would have been helpful to ascertain whether any participants had existing eye diseases, ${ }^{3}{ }^{4}$ as this could diminish their response to light therapy. Ophthalmic dysfunctions might weaken light's ability to promote positive moods.

Girardin Jean-Louis, PhD SUNY Downstate Medical Center (Departments of Ophthalmology and Psychiatry), Brooklyn, NY, USA

1 Tuunainen A, Kripke DF, Endo T. Light therapy for non-seasonal depression. Cochrane Database Syst Rev 2004; CD004050.

2 Golden RN, GaynesBN, Ekstrom RD, et al. The efficacy of light therapy in the treatment of mood disorders: a review and meta-analysis of the evidence. Am J Psychiatry 2005;162:656-62.

3 Jean-Lovis G, Kripke D, Cohen C, et al. Associations of ambient illumination with mood: contribution of ophthalmic dysfunctions. Physiol Behav 2005;84:479-87.

4 Fountoulakis $K N$, Fotiou $F$, lacovides $A$, et al. Is there a dysfunction in the visual system of depressed patients? Ann Gen Psychiatry 2005;4:7. 